in $S$. lineatus using a sterile-male technique, in which irradiated sperm competes with normal sperm for 'fertilizing' eggs, but after 'fertilization' no embryo develops. By irradiating males with $15 \mathrm{krad} \gamma$-radiation from a ${ }^{60} \mathrm{Co} \gamma$-emitter (Department of Nuclear Engineering, Ben Gurion University), sperm were made sterile without affecting male performance. Females were paired with two males in all combinations of normal $(\mathrm{N})$ and sterile (S) males: NN $(n$ $=9+13$ from the previous year), $\mathrm{SS}(n=$ $10)$, SN $(n=14)$ and NS $(n=10)$. None of the eggs in the SS matings hatched. We corrected the hatching success of clutches from $\mathrm{SN}$ and NS matings by the proportion of unhatched eggs in the NN controls $(13.6 \%)$. There was no first-male priority. Indeed, the calculated probabilities of fertilization by first (P1) and second (P2) males to mate suggest a complete mixing of sperm: $\mathrm{P} 1(\mathrm{SN})=0.417, \mathrm{P} 1(\mathrm{NS})=0.607$, $\mathrm{P} 2(\mathrm{SN})=0.583$ and $\mathrm{P} 2(\mathrm{NS})=0.393$. Sterile sperm had a slightly lower fertilization probability than normal sperm, but this was the case regardless of whether the first or the second male was sterile.

Complete sperm mixing in the spermatheca means that a male can expect a similar fertilization success in matings with females with or without eggs. The adaptive value of male infanticide in these spiders is that males increase their reproductive success at the expense of males that matured earlier in the season, although at a cost to the females. For males, infanticide is always advantageous because their reproductive success increases with the number of matings, which is perhaps the only option for late-maturing males. But there is a risk. For females that lose their clutch to an infanticidal male, there are costs of lower survival and reduced fecundity (our unpublished data). Also, the offspring of females who raise a replacement clutch disperse late in the season and have less time to grow before the abundance of flying insects decreases. Smaller juveniles have a lower probability of survival ${ }^{11}$. This may

1. Clutton-Brock, T. H. \& Parker, G. A. Anim. Behav. 49, 1345-1365 (1994)

2. Hrdy, S. B. Ethol. Sociobiol. 1, 13-40 (1979).

3. Bertram, B. in Growing Points in Ethology. (eds Bateson, P. G. \& Hinde, R. A.) 281-301 (Cambridge Univ. Press, 1976).

4. Rohwer, S. in Current Ornithology Vol. 3 (ed. Johnston, R. F.) 353-391 (Plenum, New York, 1976)

5. Elgar, M. A. \& Crespi, B. J. (eds) in Cannibalism: Ecology and Evolution among Diverse Taxa 1-12 (Oxford Univ. and Evolution

6. Andrade, M. Science 271, 70-72 (1996)

7. Vollrath, F. \& Parker, G. A. Nature 360, 156-159 (1992)

8. Schneider, J. M. Insect Soc. 42, 237-248 (1995)

9. Evans, T. et al. Nature 376, 299 (1995).

10. Austad, S. N. in Sperm Competition and the Evolution of Animal Mating Systems (ed. Smith, R. L.) 223-249 Animal Mating Systems (ed.
(Academic, London, 1984).

(Academic, London, 1984).
Schneider, J. M. thesis, Univ. München (1992).

12. Parker, G. A. in Sexual Selection and Reproductive Competition in Insects (eds Blum, M. S. \& Blum, N. A.) 123-166 (Academic, New York, 1979).

*Also at: Max-Planck-Institut für Verhaltensphysiologie, Seewiesen, D-82319 Starnberg, Germany.

also be a cost for the male which is expressed in the reduced fitness of his offspring. However, a male that matures late in the season will have late-dispersing offspring regardless of whether he mates with a female with eggs or a virgin female.

Infanticidal males of the spider $S$. lineatus gain matings, but decrease female survival and fecundity. This male behaviour can persist only if the costs to the male's own offspring are smaller than the direct benefits to him ${ }^{12}$. Male infanticide has not been observed previously in spiders, nor

\title{
Protein-RNA molecular recognition
}

SIR - The non-canonical $\mathrm{G} \bullet \mathrm{U}$ wobble pair is an important secondary structural feature of several RNA helices. Although connected by two hydrogen bonds, $\mathrm{G} \bullet \mathrm{U}$ differs in its geometry from a Watson-Crick base pair. It participates in both protein-RNA ${ }^{1,2}$ and RNA-RNA ${ }^{3}$ interactions. The $\mathrm{G} \bullet \mathrm{U}$ pair found in the acceptor helix of Escherichia coli alanyltransfer RNA (tRNA ${ }^{\mathrm{Ala}}$ ) has recently been found to contribute in vivo to the interaction of this RNA with the enzyme alanyltRNA synthetase, by introducing a helical distortion ${ }^{1}$. This result challenges previous in vitro studies which concluded that the role of the $\mathrm{G} \cdot \mathrm{U}$ base pair in tRNA ${ }^{\mathrm{Ala}}$ is to allow alanyl-tRNA synthetase to recognize directly the 2-amino and 2'-hydroxyl groups in the minor groove of the A-form RNA helix ${ }^{2}$. But, based on recent studies into the recognition of tRNA ${ }^{\text {Gln }}$ by $E$. coli glutaminyl-tRNA synthetase ${ }^{4}$, we suggest that this discrepancy can be reconciled by considering the diverse in vivo and in vitro conditions in these two studies.

The in vitro studies, which involved quantification of the alanylation of RNA molecules with various substitutions of $\mathrm{G} \bullet \mathrm{U}$, were performed at a subsaturating concentration of alanine $(22 \mu \mathrm{M}$; Michaelis constant, $K_{\mathrm{m}}=240 \mu \mathrm{M}$; ref. 5) due to inherent limitations of the assay system. Subsequent analysis of these experiments assumed that the use of diverse RNA substrates would not affect the kinetic parameters of alanyl-tRNA synthetase for alanine. But this critical assumption may not be valid, as it has now been shown for the glutaminyl- and tryptophanyltRNA synthetases that the mutation of certain nucleotides in the tRNA leads to substantial changes in the kinetic parameters of these synthetases with respect, not only to the tRNA, but also to the amino-acid substrate ${ }^{6}$. The replacement of $\mathrm{G} 3 \cdot \mathrm{C} 70$ by $\mathrm{G} \cdot \mathrm{U}$ in the acceptor helix of tRNA $^{\mathrm{Gln}}$, for example, leads to an almost threefold increase in the $K_{\mathrm{m}}$ for glutamine but no significant change in the catalytic constant, $k_{\text {cat }}$. If a comparable situation were to exist during the recognition of tRNA $^{\text {Ala }}$ variants by alanyl-tRNA synthetase, use of a uniform subsaturating to our knowledge in other invertebrates. However, sexually selected infanticide may be more common than previously recognized. We predict that male infanticidal strategies will be found in more invertebrate species that exhibit extreme maternal brood care.

Jutta M. Schneider*, Yael Lubin

Mitrani Center of Desert Ecology,

Jacob Blaustein Institute of

Desert Research,

Ben Gurion University of the Negev,

84990 Sede Boker, Israel

concentration of alanine would lead to non-uniform underestimation of $k_{\text {cat }}$, and consequently to the misassignment of energetic contributions to binding.

In contrast, the in vivo assay of this protein-RNA interaction is less susceptible to such errors, because the concentration of alanine in the amino-acid pool of $E$. coli is almost an order of magnitude higher than used in vitro (168 $\mu \mathrm{M}$; ref. 7). Nevertheless, the in vivo assay also suffers from a limitation in that the concentration of alanyltRNA synthetase may depend on several metabolic parameters, leading to the possibility that the observed differences in tRNA alanylation may be due to changes in the level of the enzyme itself, rather than changes in the structure of tRNA ${ }^{\text {Ala }}$. Add to this the possibility that alanyl-tRNA synthetase undergoes tRNA-dependent changes in its kinetic parameters for alanine, and what initially seem to be contradictory results on the recognition of $G \bullet U$ by alanyl-tRNA synthetase can, in fact, be readily reconciled. In this way, a nucleotide replacement that substantially reduces the level of tRNA charging under certain conditions in vitro may be effectively compensated for in vivo by increased concentrations of both amino acid and synthetase, particularly when the gene encoding alanyl-tRNA synthase is overexpressed ${ }^{1}$.

Although the problem in interpreting these results raises the perennial concern of the validity of comparing in vitro and in vivo approaches to studying molecular recognition, it shows that in this case the question of direct or indirect recognition will finally be resolved only by determining the crystallographic structure of tRNA ${ }^{\mathrm{Ala}}$ complexed with alanyl-tRNA synthetase.

Michael Ibba, Dieter Söll

Department of Molecular Biophysics and Biochemistry,

Yale University, 266 Whitney Avenue, New Haven, Connecticut 06520-8114, USA

1. Gabriel, K. et al. Science 271, 195-197 (1996)

2. Musier-Forsyth, K. \& Schimmel, P. Nature 357, 513-515 (1992)

3. Strobel, S. A. \& Cech, T. R. Science 267, 675-679 (1995)

4. Hong, K.-W. et al. EMBO J. 15, 1983-1991 (1996).

5. Shi, J.-P. et al. Biochemistry 33, 5312-5318 (1994).

6. Ibba, M. et al. Proc. natn. Acad. Sci. U.S.A. (in the press)

7. Raunio, R. \& Rosenqvist, H. Acta chem. scand. 24, 2737 (1970). 